\title{
Estudo da dilatação volumétrica do óleo bruto de castanha de caju: predição do coeficiente de expansão térmica
}

César Augusto Canciam, Mestre em Engenharia Química, professor lotado no Departamento Acadêmico de Engenharia Química da Universidade Tecnológica Federal do Paraná - Câmpus Ponta Grossa, canciam@utfpr.edu.br

Recebido em: 28/05/2013 - Aprovado em: 15/07/2013 - Disponibilizado em: 15/08/2013 RESUMO:

O estudo da dilatação térmica em líquidos é feito somente em relação à dilatação volumétrica, sendo importante o conhecimento do coeficiente de expansão térmica. O objetivo deste trabalho foi predizer o coeficiente de expansão térmica do óleo bruto de castanha de caju a partir da modelagem matemática dos dados experimentais da densidade em função da temperatura. Para tanto, foram utilizadas as modelagens propostas por ALVARADO (1995) e JERÔNIMO (2012). As duas modelagens matemáticas propõem a análise de regressão linear como forma de obtenção do coeficiente de expansão térmica. Os valores obtidos para o coeficiente de expansão térmica do óleo bruto de castanha de caju

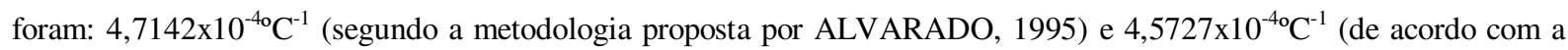
metodologia proposta por JERÔNIMO, 2012). Nas duas metodologias, os coeficientes de correlação foram próximos da unidade. Não foram encontrados valores experimentais para o coeficiente de expansão térmica do óleo bruto de castanha de caju na literatura.

PALAVRAS-CHAVE: Dilatação. Óleo. Castanha. Caju. Predição.

\section{The study of volumetric expansion of the crude cashew chestnut oil: prediction of the thermal expansion coefficient}

\section{ABSTRACT:}

The study of the thermal dilation in liquids is only made in relation to the volumetric dilation, but it is also important to know the thermal expansion coefficient. The objective of this work was to predict the thermal expansion coefficient of the crude cashew chestnut oil from the mathematical modeling of the experimental data of density as a function of temperature. For this, the mathematical models used were proposed by ALVARADO (1995) and JERÔNIMO (2012). The two mathematical models propose the linear regression analysis in order to obtain the thermal expansion coefficient. The values obtained for the thermal expansion coefficient of the crude cashew chestnut oil were: $4.7142 \times 10^{-}$ ${ }^{40} \mathrm{C}^{-1}$ (according to the methodology proposed by ALVARADO, 1995) and $4.5727 \times 10^{-40} \mathrm{C}^{-1}$ (according to the methodology proposed by JERÔNIMO, 2012). In both methods, the correlation coefficients were close to unity. There were no experimental values for the thermal expansion coefficient of the crude cashew chestnut oil in the literature.

KEYWORDS: Dilation. Oil. Chestnut. Cashew. Prediction.

\section{INTRODUÇÃO}

A dilatação de um material em decorrência da elevação da temperatura é uma consequência do aumento da sua energia interna, que determina uma maior amplitude das vibrações moleculares e, portanto, uma maior distância média entre os constituintes moleculares (FRADE, 2010). 
O coeficiente de expansão térmica $(\beta)$

é uma propriedade volumétrica que está relacionada com a resposta de um material à aplicação de calor (AMORIM, 2007; CALLISTER, 2008).

Juntamente com a compressibilidade isotérmica e o volume de excesso, o coeficiente de expansão térmica é uma propriedade volumétrica derivada da

densidade $(\rho)$, sendo definida conforme a Equação 1 (SINGH et al., 2006; AMORIM, 2007).

$$
\beta=\frac{1}{V} \cdot\left(\frac{\partial V}{\partial T}\right)_{P}=-\frac{1}{\rho} \cdot\left(\frac{\partial \rho}{\partial T}\right)_{P}
$$

Em que $V$ corresponde ao volume; $T$,

à temperatura e $P$, à pressão.

O conhecimento do coeficiente de expansão térmica permite (YOUNG e FREEDMAN, 2008; SANTOS e VIEIRA, 2010; JERÔNIMO et al., 2012; CANCIAM, 2012):

- Estudar o comportamento da dilatação do material;

- Avaliar os impactos no sistema de medição volumétrico decorrentes da variação de temperatura;

- Servir de dado no projeto de equipamentos e acessórios considerando possíveis dilatações advindas das fortes variações de temperatura;

- Corresponder a uma das variáveis na estimativa de outras propriedades, dentre elas: a tensão volumétrica e a entalpia de vaporização;

- Estudar a convecção livre que influencia na transferência de calor em tubulações.

A dilatação volumétrica é variável e tende a manter uma relação direta com a composição química dos diferentes materiais líquidos (JERÔNIMO et al., 2012).

CANCIAM (2008), SANTOS e VIEIRA (2010) e JERÔNIMO (2012) comentam que os valores do coeficiente de expansão térmica estão relacionados com a energia de ligação química entre as espécies químicas. Materiais em que as ligações químicas são fortes apresentam baixos coeficientes de expansão térmica. Isto porque a dilatação térmica está associada à variação assimétrica da energia de ligação com a distância entre as espécies químicas. Ou seja, durante o aquecimento as espécies químicas do material aumentam a frequência e a amplitude de vibração (estado excitado) e como as forças de repulsão são sempre maiores que as forças de atração, a distância entre as espécies químicas também aumenta.

O cajueiro (Anacardium occidentale, L.) é cultivado em vários países, destacando: Brasil, Índia, Moçambique, Tanzânia, Quênia, 
Guiné Bissau, Benin, Indonésia, Vietnã e Tailândia (LIMA, 2009).

A mesma autora comenta que no Brasil, o cajueiro é disseminado em todo o território. Entretanto, em termos de importância econômica, sua exploração concentra-se nos estados do Ceará, Piauí e Rio Grande do Norte.

A amêndoa da castanha de caju é um dos principais produtos de utilização do cajueiro. O principal componente da amêndoa da castanha de caju são os lipídios (46,28\% na amêndoa crua e 48,35\% na amêndoa tostada) (GAZZOLA et al., 2006).

Com relação aos lipídios presentes na amêndoa da castanha de caju, os mesmos autores comentam que, em termos de ácidos graxos, estão presentes os ácidos: linoleico (até 21,7\%), esteárico (1,5 a 11,2\%), palmítico $(4,1$ a $17,3 \%)$ e oleico $(68,2$ a $80,4 \%)$.

Se considerar que na obtenção do óleo bruto de castanha de caju, todos os lipídios presentes na amêndoa são extraídos, é de se esperar que este óleo apresente, como principal ácido graxo, o ácido oleico.

O ácido oleico ou ácido cis-9octadecenóico é considerado um ácido graxo monoinsaturado constituído por dezoito átomos de carbono com uma dupla ligação começando no nono átomo de carbono contado a partir do átomo de carbono do grupo metila (BRUICE, 2006).

Quanto à utilização do óleo de castanha de caju, GAZZOLA et al. (2006) relatam da utilização do óleo de castanha de caju como substituto do azeite de oliva na alimentação humana.

Já RODRIGUES et al. (2010) comentam da utilização do óleo bruto de castanha de caju como óleo lubrificante, em virtude de suas propriedades.

O objetivo deste trabalho foi predizer o coeficiente de expansão térmica do óleo bruto de castanha de caju a partir da modelagem matemática de dados experimentais da densidade em função da temperatura. Para tanto, foram consideradas as metodologias propostas por ALVARADO (1995) e JERÔNIMO (2012).

\section{MATERIAIS E MÉTODOS}

A Tabela 1 relaciona os dados experimentais da densidade $(\rho)$ em função da temperatura $(\mathrm{T})$ para o óleo bruto de castanha de caju. Esses dados foram adaptados do trabalho de RODRIGUES et al. (2010).

Tabela 1 - Efeito da temperatura na densidade do óleo bruto de castanha de caju

\begin{tabular}{cc}
\hline Temperatura $\left({ }^{\circ} \mathrm{C}\right)$ & Densidade $\left(\mathrm{kg} \cdot \mathrm{m}^{-3}\right)$ \\
\hline 26 & 985,6407 \\
40 & 980,4437 \\
60 & 972,6568 \\
80 & 963,1367 \\
100 & 952,7572 \\
Fonte: & Adaptado de RODRIGUES et al. $(2010)$.
\end{tabular}

A metodologia proposta por ALVARADO (1995) considera a análise de 
regressão linear de dados de $\frac{1}{\rho}$ em função da temperatura $(\mathrm{T})$ para a obtenção do coeficiente de expansão térmica $(\beta)$. A Equação 2 relaciona a modelagem matemática proposta por ALVARADO (1995).

$$
\frac{1}{\rho}=\frac{1}{\rho^{*}}+\left(\frac{\beta}{\rho^{*}}\right) \cdot \mathrm{T}
$$

Em que $\rho^{*}$ corresponde a uma constante característica para cada óleo vegetal.

A metodologia proposta por JERÔNIMO (2012) considera a análise de regressão linear de dados de $\ln \left(\frac{\rho_{0}}{\rho}\right)$ em função de $\left(\mathrm{T}-\mathrm{T}_{0}\right)$ para a obtenção do coeficiente de expansão térmica. A Equação 3 relaciona a modelagem matemática proposta por JERÔNIMO (2012).

$$
\ln \left(\frac{\rho_{0}}{\rho}\right)=\beta \cdot\left(\mathrm{T}-\mathrm{T}_{0}\right)
$$

Em que $\rho_{0}$ corresponde à densidade na temperatura $\mathrm{T}_{0}$.

Aplicando os dados experimentais da Tabela 1 na Equação 2 (modelagem matemática proposta por ALVARADO, 1995) e na Equação 3 (modelagem matemática proposta por JERÔNIMO, 2012) foi possível predizer o coeficiente de expansão térmica para o óleo bruto de castanha de caju.
Cada uma das análises de regressão linear foi realizada utilizando um programa específico de análise de regressão linear disponível na calculadora CASIO FX-850P Scientific Library 116.

Nos cálculos para o modelo de JERÔNIMO (2012), considerou-se a temperatura $\mathrm{T}_{0}$ como sendo $26^{\circ} \mathrm{C}$ e a densidade $\rho_{0}$ equivalente a $985,6407 \mathrm{~kg} \cdot \mathrm{m}^{-3}$.

\section{RESULTADOS E DISCUSSÃO}

A Figura 1 ilustra o gráfico de $\frac{1}{\rho}$ versus $\mathrm{T}$ para o óleo bruto de castanha de caju.

Figura 1: Gráfico $(1 / \rho)$ versus $\mathrm{T}$ para o óleo bruto de castanha de caju

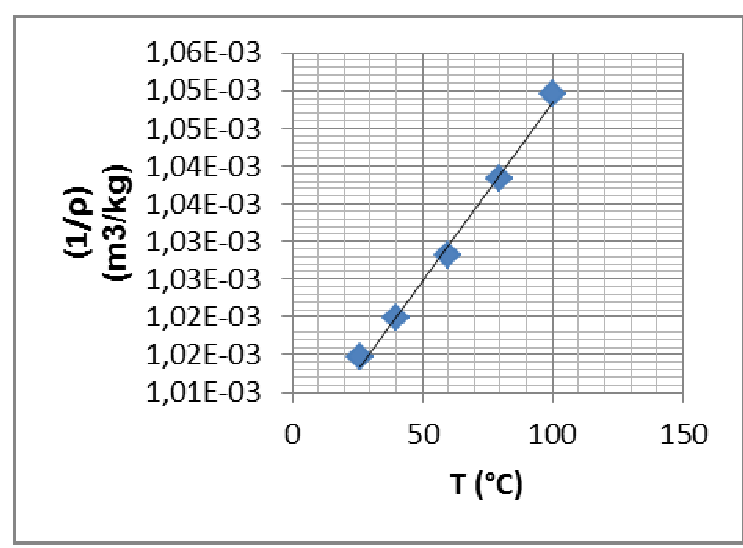

Fonte: Autor

A Tabela 2 relaciona os resultados obtidos da análise de regressão linear de $\frac{1}{\rho}$ em função da temperatura $(\mathrm{T})$, de acordo com a metodologia proposta por ALVARADO (1995). 
Com base nos resultados da Tabela 2 , a constante $\rho^{*}$ equivale a $998,8014 \mathrm{~kg} . \mathrm{m}^{-3}$, o coeficiente de expansão térmica $(\beta)$, a $4,7142 \times 10^{-4} \mathrm{C}^{-1}$ e o coeficiente de correlação $\left(\mathrm{R}^{2}\right)$ equivale a 0,9967 .

Tabela 2 - Resultados da análise de regressão linear de acordo com a modelagem matemática proposta por

\begin{tabular}{cc}
$\frac{1}{\rho^{*}}$ & $1,0012 \times 10^{-3} \mathrm{~m}^{3} \cdot \mathrm{kg}^{-1}$ \\
$\frac{\beta}{\rho^{*}}$ & $4,7199 \times 10^{-7}$ \\
$\mathrm{R}^{2}$ & 0,9967 \\
\hline
\end{tabular}

Fonte: Autor.

Não foram encontrados valores para $\frac{1}{\rho^{*}}$, considerando o óleo bruto de castanha de caju.

ALVARADO (1995), estudando a dilatação volumétrica de alguns óleos vegetais brutos entre 20 e $70^{\circ} \mathrm{C}$, obteve para $\frac{1}{\rho^{*}}$, valores que variaram de $1,031 \times 10^{-3}$ a $1,076 \times 10^{-3} \mathrm{~m}^{3} \cdot \mathrm{kg}^{-1}$.

Observa-se que o valor de $\frac{1}{\rho^{*}}$ encontrado para o óleo bruto de castanha de caju está fora deste intervalo.

Como comentado anteriormente, a constante $\rho^{*}$ é característica de cada óleo vegetal.

Com o valor encontrado $\left(1,0012 \times 10^{-3}\right.$ $\left.\mathrm{m}^{3} \cdot \mathrm{kg}^{-1}\right)$, sugere-se que os valores de $\frac{1}{\rho^{*}}$, para qualquer óleo vegetal bruto, sejam da ordem de $1,0 \times 10^{-3}$. Isto é apenas uma sugestão, que necessita ser mais investigada com outros óleos vegetais brutos.

A Figura 2 ilustra o gráfico de $\ln \left(\frac{\rho_{0}}{\rho}\right)$ versus $\left(\mathrm{T}-\mathrm{T}_{0}\right)$ para o óleo bruto de castanha de caju.

Figura 2: Gráfico $\ln \left(\frac{\rho_{0}}{\rho}\right)$ versus $\left(\mathrm{T}-\mathrm{T}_{0}\right)$ para o óleo bruto de castanha de caju

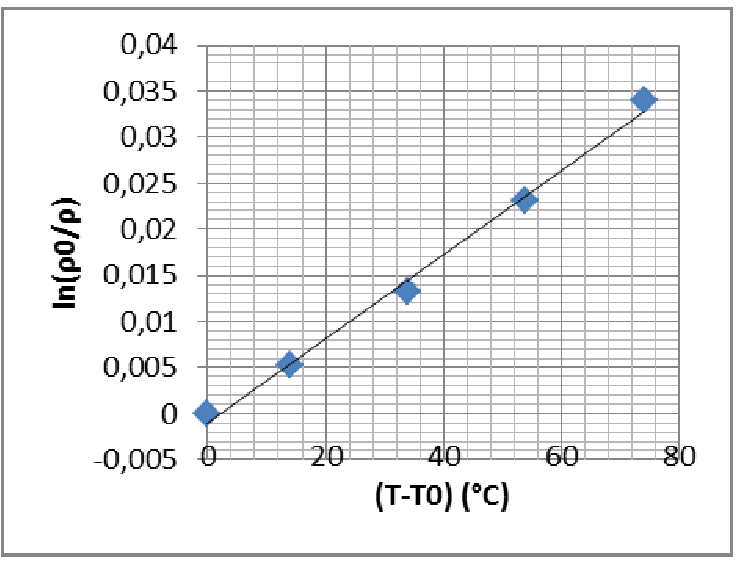

Fonte: Autor

A Tabela 3 relaciona os resultados obtidos da análise de regressão linear de $\ln \left(\frac{\rho_{0}}{\rho}\right)$ em função de $\left(T-T_{0}\right)$, de acordo com a metodologia proposta por JERÔNIMO (2012).

Tabela 3 - Resultados da análise de regressão linear de acordo com a modelagem matemática proposta por

$$
\text { JERÔNIMO (2012) }
$$

\begin{tabular}{cc}
\hline$\beta$ & $4,5727 \times 10^{-4 o} \mathrm{C}^{-1}$ \\
$\mathrm{R}^{2}$ & 0,9972 \\
\hline
\end{tabular}

Fonte: Autor. 
Com relação ao coeficiente de correlação $\left(\mathrm{R}^{2}\right)$, PINHEIRO et al. (2009) comentam que este parâmetro mede a interdependência linear entre as variáveis e avalia a qualidade do ajuste, ou seja, quanto mais próximo o coeficiente de correlação for da unidade, melhor o ajuste da reta em relação aos pontos da dispersão.

LIRA (2004) fornece uma classificação para as correlações lineares. A autora comenta que a correlação linear é classificada como muito forte quando os valores do coeficiente de correlação $\left(\mathrm{R}^{2}\right)$ são maiores ou iguais a 0,90 e menores que 1,0 .

Dessa forma, observa-se na Tabela 2 e na Tabela 3, que para as metodologias adotadas na predição do coeficiente de expansão térmica do óleo bruto de castanha de caju, a correlação linear é classificada como muito forte.

Quanto aos valores do coeficiente de expansão térmica, a literatura não dispõe de valores experimentais para o óleo bruto de castanha de caju.

CANCIAM (2011), estudando a dilatação volumétrica do óleo bruto de mamona das cultivares BRS-149 Nordestina e BRS-188 Paraguaçu, encontrou para o coeficiente de expansão térmica, valores iguais a $5,2745 \times 10^{-4 o} \mathrm{C}^{-1}$ (cultivar BRS-149 Nordestina) e $5,2705 \times 10^{-4} \mathrm{C}^{-1}$ (cultivar BRS188 Paraguaçu). O intervalo de temperatura é de -15 a $80^{\circ} \mathrm{C}$.
Comparando os valores encontrados neste trabalho com os valores encontrados por CANCIAM (2011) para o óleo bruto de mamona, a diferença entre os coeficientes de expansão térmica do óleo bruto de mamona com o óleo bruto de castanha de caju variam de $5,563 \times 10^{-5}$ a $7,018 \times 10^{-5 o} \mathrm{C}^{-1}$. Dessa forma, os resultados sugerem que o coeficiente de expansão térmica do óleo bruto de castanha de caju está próximo ao coeficiente de expansão térmica do óleo bruto de mamona.

O coeficiente de expansão térmica mede a variação relativa do volume devido à alteração da temperatura, mantendo-se a pressão constante. Assim, por exemplo, se o coeficiente de expansão térmica de um material é igual a $1,0 \times 10^{-2 o} \mathrm{C}^{-1}$ significa que o acréscimo de 1 grau tem como resultado o aumento do volume em 1\% (NETZ e ORTEGA, 2008).

Assim, seguindo a mesma linha de raciocínio, para cada 1 grau de acréscimo de temperatura, o aumento do volume do óleo bruto de castanha de caju varia de 0,046 a $0,047 \%$.

Estes valores podem parecer pequenos, mas se comparados com a água, o aumento do volume do óleo bruto de castanha de caju equivale 2,21 a 2,28 vezes mais que o aumento do volume da água.

Nos cálculos acima citados, foi considerado que o coeficiente de expansão térmica da água equivale a $2,07 \times 10^{-40} \mathrm{C}^{-1}$ (CABRAL e LAGO, 2002). 
A água apresenta interações intermoleculares do tipo ligações de hidrogênio. Enquanto que os óleos vegetais apresentam interações intermoleculares do tipo forças de dispersão de London (CANCIAM, 2010).

NETZ e ORTEGA (2008) comentam que as ligações de hidrogênio são mais fortes em comparação às forças de dispersão de London. Isto justificaria o menor valor do coeficiente de expansão térmica da água em relação ao coeficiente de expansão térmica do óleo bruto de castanha de caju.

\section{CONCLUSÃO}

A metodologia proposta por ALVARADO (1995) obteve, para o coeficiente de expansão térmica $(\beta)$ do óleo bruto de castanha de caju, o valor de $4,7142 \times 10^{-4}{ }^{\circ} \mathrm{C}^{-1}$. Enquanto que a metodologia proposta por JERÔNIMO (2012), o valor obtido equivale a $4,5727 \times 10^{-4 o} \mathrm{C}^{-1}$. A faixa de temperatura, considerada para ambos os casos, é de 26 a $100^{\circ} \mathrm{C}$.

Para as duas metodologias aplicadas, as correlações lineares foram classificadas como muito fortes.

A cada 1 grau de acréscimo de temperatura, o aumento do volume do óleo bruto de castanha de caju varia de 0,046 a $0,047 \%$.
Verificou-se a ausência na literatura de valores do coeficiente de expansão térmica para o óleo bruto de castanha de caju.

Os valores do coeficiente de expansão térmica do óleo bruto de castanha de caju preditos neste trabalho foram próximos aos valores do coeficiente de expansão térmica do óleo bruto de mamona.

\section{REFERÊNCIAS}

ALVARADO, J. D. Propiedades mecânicas de aceites y grasas vegetales. Grasas y Aceites, v. 46, n. 4-5, p. 264-269, 1995.

AMORIM, J. A. Obtenção de propriedades físico-químicas de misturas de hidrocarbonetos em baixa e alta pressão visando a caracterização e a modelagem. Tese de Doutorado em Engenharia Química da Universidade Federal do Rio Grande do Norte, 2007. 165p.

BRUICE, P. Y. Química Orgânica - volume 2. Pearson Education do Brasil, São Paulo, 2006. 641p.

CABRAL, F.; LAGO, A. Física 2. Harbra, São Paulo, 2002. 516p.

CALLISTER, W. D. Ciência e Engenharia de Materiais: uma introdução. LTC, Rio de Janeiro, 2008. 590p.

CANCIAM, C. A. Predição do coeficiente de 
expansão térmica do óleo de soja (Glicine max). Tecnológica, v. 17, p. 13-18, 2008.

CANCIAM, C. A. Predição do coeficiente de expansão térmica do óleo de milho. Revista CIATEC, v. 2, n. 2, p. 15-21, 2010.

CANCIAM, C. A. Predição do coeficiente de expansão térmica e da energia de ativação do óleo bruto de mamona através da aplicação da análise de regressão linear. E-xacta, v. 4, n. 3, p. 7-18, 2011.

CANCIAM, C. A. Predição do coeficiente de expansão térmica do óleo de girassol através da aplicação da análise de regressão linear.

Revista Brasileira de Tecnologia

Agroindustrial, v. 6, n. 2, p. 852-863, 2012.

FRADE, J. M. C. B. C. Conformação automática de formas complexas em vidro de mesa. Tese de Doutorado em Ciência e Engenharia dos Materiais da Universidade de Aveiro, 2010. 327p.

GAZZOLA, J.; GAZZOLA, R.; COELHO, C. H. M.; WANDER, A. E.; CABRAL, J. E. O. A amêndoa da castanha-de-caju: composição e importância dos ácidos graxos - produção e comércio mundiais. In: CONGRESSO DA

SOCIEDADE BRASILEIRA DE ECONOMIA E SOCIOLOGIA RURAL, 44., 2006, Fortaleza. Anais... Fortaleza: SOBER, 2006.
JERÔNIMO, C. E. M. Predição do

coeficiente de dilatação térmica de naftas pela curva de destilação ASTM. Revista Virtual de Química, v. 4, n. 4, p. 405-412, 2012.

JERÔNIMO, C. E. M.; BALBINO, C. P.; FERNANDES, H. G. Coeficiente de dilatação volumétrica determinados pela curva ASTM em frações de petróleo. Scientia Plena, v. 8, n. 9, p. 1-8, 2012.

LIMA, E. D. Estudo do despeliculamento da amêndoa da castanha de caju com aplicação de baixas temperaturas e ultra-som.

Dissertação de Mestrado do Programa de Pósgraduação em Ciência e Tecnologia de Alimentos da Universidade Federal do Ceará, 2009. 74p.

LIRA, S. A. Análise de correlação: abordagem teórica e de construção dos coeficientes com aplicação. Dissertação de Mestrado do Programa de Mestrado em Ciências da Universidade Federal do Paraná, 2004. 196p.

NETZ, P. A.; ORTEGA, G. G. Fundamentos de físico-química: uma abordagem conceitual para as ciências farmacêuticas. Artmed, Porto Alegre, 2008. 299p.

PINHEIRO, J. I. D.; CUNHA, S. B.; CARVAJAL, S. R.; GOMES, G. C. Estatística básica: a arte de trabalhar com 
dados. $1^{\text {a }}$ ed. Elsevier, Rio de Janeiro, 2009.

$295 \mathrm{p}$.

RODRIGUES, L. K. O.; CAVALCANTE, S.

L. L.; LIMA, R. S.; RIBEIRO, F. A.;

MENDES, J. U. L. Análise de propriedades

intensivas do óleo da castanha de caju.

PublICa, v. VI, p. 30-36, 2010.

SANTOS, D. Q.; VIEIRA, D. F.

Determinação de coeficiente de expansão

térmica do biodiesel e seus impactos no

sistema de medição volumétrico. Eclética

Química, v. 35, n. 4, p. 107-112, 2010.

SINGH, R. N.; GEORGE, A. K.; ARAFIN, S.

Specific heat ratio, Grüneisen parameter and

Debye temperature of crude oil. Journal of

Physics D: Applied Physics, v. 39, p. 1220-

$1225,2006$.

YOUNG, H. D.; FREEDMAN, R. A. Física

III- Termodinâmica e Ondas. $12^{\mathrm{a}} \mathrm{ed}$. Addison

Wesley, São Paulo, 2008. 318p. 